\title{
Are we Improving Adherence to Cardiovascular Guidelines?
}

\author{
Luciana Nicolau Aranha ${ }^{\circledR}$ and Gláucia Maria Moraes de Oliveira \\ Universidade Federal do Rio de Janeiro, Rio de Janeiro, RJ - Brazil \\ Editorial related to the article: Treatment Eligibility and Therapeutic Responses of an Ecuadorian Population at High Cardiovascular Risk Based on \\ the ATP III Guidelines
}

Chronic noncommunicable diseases (NCDs) comprise the world's leading group of causes of death, accounting for premature deaths, loss of quality of life, and adverse economic and social impacts. They represent about 70\% of global deaths, close to 38 million deaths annually, significantly exceeding deaths from external causes and infectious diseases, such as COVID-19. Almost $45 \%$ of the deaths, more than 17 million, occur as a result of cardiovascular disease (CVD). ${ }^{1}$ The same happens in Brazil, where $72 \%$ of the deaths are due to NCDs, and about $30 \%$, to CVD. ${ }^{1}$

Dyslipidemia is a significant risk factor for CVD, particularly coronary artery disease (CAD) and stroke. Prospective and long-term epidemiological studies have shown that individuals with healthier lifestyles and fewer risk factors for CAD could improve their life expectancy by decreasing cardiovascular morbidity and mortality. ${ }^{2}$ The Framingham Offspring Study, which followed 3501 participants from 1987 to 2011, has demonstrated that low levels of high-density lipoprotein (HDL) or high levels of low-density lipoprotein (LDL) and triglycerides, alone or in any combination, are associated with increased risk for CVDs. ${ }^{3}$ Therefore, intervention measures are necessary to reduce morbidity and mortality, as well as the cost of hospitalization due to those diseases. In addition, improving the quality of life and health of the population is essential.

Lifestyle changes, such as the adoption of a healthy diet and physical activity, can favorably affect plasma

\section{Keywords}

Cardiovascular Diseases/complications, Mortality; Cardiovascular Diseases/prevention and control; COVID-19; Dyslipidemias; Triglycerides; Life Style; Tabagism; Hydroxymethylglutaryl-CoA Reductase Inhibition/therapeutic use; Patient Compliance. lipid concentrations. However, many patients need medication to achieve their therapeutic goals. ${ }^{2}$ In recent years, lipid-lowering therapy, in particular statins, has been one of the most used interventions for primary and secondary prevention of atherosclerotic cardiovascular diseases, since studies have shown their efficacy in reducing both future events and CVD mortality. ${ }^{4,5}$

In this sense, to identify individuals at risk for CVD, guidelines have been developed, recommending the use of lipid-lowering therapy for the prevention/treatment of those diseases. The National Cholesterol Education Program-Adult Treatment Panel III (NCEP-ATP III) used the Framingham score to assess the risk of CAD for ten years. The NCEP-ATP III classified individuals into three categories, high risk $(>20 \%)$, moderate risk $(10-20 \%)$ and low risk $(<10 \%)$, suggesting the use of lipid-lowering therapy to reduce LDL $(<100 \mathrm{mg} / \mathrm{dl})$ in patients at high risk for CAD, whose LDL concentrations are $\geq 130 \mathrm{mg} / \mathrm{dl}$. The HDL targets were considered secondary objectives in patients with high levels of triglycerides (> $200 \mathrm{mg} / \mathrm{dl}) .{ }^{6}$

An observational and retrospective study, evaluating the adherence of internal medicine residents in Worcester, Massachusetts, to the NCEP-ATP III guidelines for the treatment of patients with dyslipidemia, has shown better adherence to drug therapy $(44 \%-77 \%)$ and changes in lifestyle $(44 \%-83 \%)$ as compared to the recommendations for follow-up $(22 \%-31 \%)$, evidencing the difficulties of adherence over time. ${ }^{7}$

The American College of Cardiology (ACC) and the American Heart Association (AHA) developed in 2013 a guideline on cardiovascular risk assessment and hypercholesterolemia management. The guideline established new perspectives on LDL treatment goals, using a new tool to assess cardiovascular risk, the pooled cohort equations (PCE). The guideline suggested the use of statin to individuals aged 40 to 75 years, without

Mailing Address: Gláucia Maria Moraes de Oliveira

Universidade Federal do Rio de Janeiro - R. Prof. Rodolpho P. Rocco, 255 - 8. Andar - Sala 6, UFRJ. Postal Code: 21941-913, Cidade Universitária, RJ - Brazil.

E-mail: glauciam@cardiol.br, glauciamoraesoliveira@gmail.com 
a diagnosis of clinical atherosclerotic cardiovascular disease or diabetes mellitus, with LDL-c between $70 \mathrm{mg} / \mathrm{dl}$ and $189 \mathrm{mg} / \mathrm{dl}$ and cardiovascular risk by PCE $\geq 7.5 \%$ in 10 years. $^{8}$ In 2018, a new AHA/ACC cholesterol guideline was published, proposing the categorization of cardiovascular risk and recommending the start of statin therapy for individuals at intermediate or high risk and considering those at borderline risk in certain circumstances. The use of ezetimibe and PCSK9 inhibitors was also suggested for those with severe primary hypercholesterolemia. ${ }^{9}$

In the current edition of the International Journal of Cardiovascular Sciences, Hernandez et al., ${ }^{10}$ have analyzed lipid-lowering regimens in Ecuadorian patients to assess whether the therapy targets of the NCEP-ATP III guidelines were achieved. Those authors carried out a retrospective analysis with 385 patients (46\% men; mean age, $59.8 \pm 13.2$ years). They observed that $68 \%$ of the patients had a very high risk for CAD, and most of them $(n=253 ; 95,8 \%)$ were on atorvastatin $(50 \%)$, simvastatin $(34,8 \%)$, and rosuvastatin $(11 \%)$. Regarding the targets of the NCEP-ATP III guidelines, the authors observed that only 24 individuals (19\%) with high cardiovascular risk reached LDL-c $<100 \mathrm{mg} / \mathrm{dl}$, while 30 individuals $(11 \%)$ with very high risk reached LDL-c $<70 \mathrm{mg} / \mathrm{dl}$. The results were also analyzed based on the 2013 ACC/AHA guidelines, and only $10.7 \%$ of the patients receiving highintensity statins reached the target of a $50 \%$ reduction in LDL cholesterol.

Few studies have described the impact of guidelines on clinical practice. Yu et al., ${ }^{11}$ have assessed the achievement of statin-use goals, as well as LDL-c levels before and after the 2013 ACC/AHA guidelines in 1938 patients with CAD. Those authors have reported that the proportion of patients achieving LDL-c goals ranged from $51 \%$ to $56 \%$ for the $70-\mathrm{mg} / \mathrm{dl}$ target and from $77 \%$ to $85 \%$ for the $100-\mathrm{mg} / \mathrm{dl}$ target, remaining unchanged in the comparison cohorts. ${ }^{10}$

The authors have attributed those results to the prescription of low doses of drugs and to therapies aimed at isolated lipid fractions. Other factors, however, must be considered, such as the knowledge of healthcare professionals about the guidelines and adherence to the treatment proposed, which includes both drug therapy and changes in lifestyle.

In this sense, the guidelines are consistent in recommending to all individuals, regardless of their level of risk, changes in lifestyle, such as the following: adoption of a healthy eating pattern, which emphasizes the intake of fruits, vegetables, whole grains and healthy sources of protein, and limits the consumption of ultra-processed foods; maintenance of body weight; regular physical exercise; and smoking cessation. Those measures are the basis of any intervention to reduce the risk of CVD. $6,8,9,11$

Considering the multifactorial nature of CVDs, it is necessary to implement a multidisciplinary approach to the set of risk factors responsible for their occurrence, morbidity, and mortality, with guidelines that contemplate these premises. This relates directly to the success of the proposed recommendations. Thus, the successful treatment of dyslipidemias will not only depend on the degree of cardiovascular risk and lipid profile, as assessed by the authors. It also depends on interventions that promote greater knowledge about the recommendations among all involved with the care, as well as on a more comprehensive scope of nonpharmacological population measures, which will have direct implications for cardiovascular outcome. . $^{12,13}$

\section{References}

1. GBD 2017 Disease and Injury Incidence and Prevalence Collaborators. Global, regional, and national incidence, prevalence, and years lived with disability for 354 diseases and injuries for 195 countries and territories, 1990-2017: a systematic analysis for the Global Burden of Disease Study 2017. Lancet. 2018;392(10159):1789-858. doi: 10.1016/ S0140-6736(18)32279-7. Epub 2018 Nov 8. Erratum in: Lancet. 2019 Jun 22;393(10190): e44.

2. Kopin L, Lowenstein C. Dyslipidemia. Ann Intern Med.2017; 167(11):ITC81-ITC96.

3. Andersson C, Lyass A, Vasan RS, Massaro JM, D'Agostino RB Sr, Robins SJ. Long-term risk of cardiovascular events across a spectrum of adverse major plasma lipid combinations in the Framingham Heart Study. Am Heart J. 2014;168(6):878-83 e1.

4. Lu Y, Cheng Z, Zhao Y, Chang X, Chan C, Bai Y, Cheng N. Efficacy and safety of long-term treatment with statins for coronary heart disease: A Bayesian network meta-analysis. Atherosclerosis.2016 Nov;254:215-27. doi: 10.1016/j.atherosclerosis.2016.10.025

5. Yebyo HG, Aschmann HE, Kaufmann M, Puhan MA. Comparative effectiveness and safety of statins as a class and of specific statins for primary prevention of cardiovascular disease: A systematic review, meta-analysis, and network meta-analysis of randomized trials with 94,283 participants. Am Heart J. 2019 Apr; 210:18-28. doi: 10.1016/j. ahj.2018.12.007.

6. Grundy SM, Cleeman JI, Bairey Merz CN, Brewer Jr B, Clark LT, Hunninghake DB, for the Coordinating Committee of the National Cholesterol Education Program Endorsed by the National Heart, Lung, 
and Blood Institute, American College of Cardiology Foundation, and American Heart Association. Implications of Recent Clinical Trials for the National Cholesterol Education Program Adult Treatment Panel III Guidelines. Circulation. 2004;110(2):227-39.

7. Vijayakrishnan R, Kalyatanda G, Srinivasan I, Abraham GM. Compliance with the Adult Treatment Panel III guidelines for hyperlipidemia in a resident-run ambulatory clinic: a retrospective data analysis. J Clin Lipidol. 2013 Jan-Feb;7(1):43-7. doi: 10.1016/j.jacl.2012.06.004.

8. Stone NJ, Robinson JG, Lichtenstein AH, Merz NB, Blum CB, Eckel RH et al. 2013 ACC/AHA Guideline on the Treatment of Blood Cholesterol to Reduce Atherosclerotic Cardiovascular Risk in Adults: a report off the American College of Cardiology/American Heart Association Task Force on Practice Guidelines. Circulation.2014;63(25 Pt B): 2889-934.

9. Grundy SM, Stone NJ, Bailey AL, Beam C, Birtcher KK, Blumenthal RS, et al. 2018 AHA/ACC/AACVP/AAPA/ABC/ACPM/ADA/ AGS/ APhA/ ASPC/NLA/PCNA. Guideline on the Management of Blood Cholesterol: a Report of the American College of Cardiology/
American Heart Association Task Force on Clinica Practice Guidelines. Circulation.2019;139(25):e1182-e1186.Correction:e1187.

10. Hernándéz I, Estrella A, Salazar J, Duarte Y, Torres E, López C, et al Treatment Eligibility and Therapeutic Responses of an Ecudorian Population at High Cardiovascular Risk Based on the ATP III Guidelines. Int J Cardiovasc Sci. 2020; 33(4):371-376.

11. Yu S, Zolfaghari K, Rascati KL, Copeland LA, Goldley PJ, McNeal C. Guidelines impact cholesterol management. J Clin Lipidol.2019;13(3):432-42.

12. Jeffery RA, To MJ, Hayduk-Costa G, Cameron A, Taylor C, Van Zoost C, Hayden JA. Adherence to cardiovascular disease guidelines: a systematic review. BMC Fam Pract. 2015;16:147. doi: 10.1186/s12875015-0341-7.

13. Précoma DB, Oliveira GMM, Simão AF, Dutra OP, Coelho OR, Izar COM, et al. Updated Cardiovascular Prevention Guideline of the Brazilian Society Cardiology - 2019. Arq Bras Cardiol. 2019;113(4):787891. doi: $10.5935 /$ abc. 20190204 